\title{
Note on Dilogarithm Identities from Nilpotent Double Affine Hecke Algebras
}

Tomoki NAKANISHI

Graduate School of Mathematics, Nagoya University, Chikusa-ku, Nagoya, 464-8604, Japan

E-mail:nakanisi@math.nagoya-u.ac.jp

Received November 15, 2012, in final form December 22, 2012; Published online December 25, 2012 http://dx.doi.org/10.3842/SIGMA.2012.104

\begin{abstract}
Recently Cherednik and Feigin [arXiv:1209.1978] obtained several Rogers-Ramanujan type identities via the nilpotent double affine Hecke algebras (Nil-DAHA). These identities further led to a series of dilogarithm identities, some of which are known, while some are left conjectural. We confirm and explain all of them by showing the connection with $Y$-systems associated with (untwisted and twisted) quantum affine Kac-Moody algebras.
\end{abstract}

Key words: double affine Hecke algebra; dilogarithm; $Y$-system

2010 Mathematics Subject Classification: 17B37; 13F60

\section{Dilogarithm identities from Nil-DAHA}

Let $R_{n}$ be a root system of finite type and of rank $n$ with non-degenerate bilinear form ( , ), and let $\alpha_{i}$ and $\omega_{i}$ be the simple roots and the fundamental weights of $R_{n}$. The Cartan matrix $C=\left(c_{i j}\right)_{i, j=1}^{n}$ is given by $c_{i j}=2\left(\alpha_{i}, \alpha_{j}\right) /\left(\alpha_{i}, \alpha_{i}\right)$. Following [3], let $A=\left(a_{i j}\right)$ and $A^{b}=\left(a_{i j}^{b}\right)$ be the matrices with $a_{i j}=2\left(\omega_{i}, \omega_{j}\right)$ and $a_{i j}^{b}=\left(\omega_{i}, \omega_{j}\right)$, respectively. Set $\nu_{i}=\left(\alpha_{i}, \alpha_{i}\right) / 2$. Then, $\nu_{i}^{-1}\left(\alpha_{i}, \omega_{j}\right)=\delta_{i j}$, and we have

$$
\left(A^{b}\right)^{-1}=\left(c_{i j} \nu_{j}^{-1}\right)_{i, j=1}^{n} .
$$

Below we normalize the bilinear form as $\left(\alpha_{\text {short }}, \alpha_{\text {short }}\right)=2$ so that $\nu_{i} \in\{1,2,3\}$.

Let $L(x)$ be the Rogers dilogarithm function

$$
L(x)=-\frac{1}{2} \int_{0}^{x}\left\{\frac{\log (1-y)}{y}+\frac{\log y}{1-y}\right\} \mathrm{d} y .
$$

In [3, equation (3.34)] Cherednik and Feigin presented two (partially conjectural) series of dilogarithm identities. Let $A^{\prime}=\left(a_{i j}^{\prime}\right)_{i, j=1}^{n}$ be either $A$ or $A^{b}$ as above. Let $Q_{i}(i=1, \ldots, n)$ be the unique solution of the system of equations

$$
\left(1-Q_{i}\right)^{\nu_{i}}=\prod_{j=1}^{n} Q_{j}^{a_{i j}^{\prime}}
$$

in the range $0<Q_{i}<1$. Then, the following identity was proposed

$$
\frac{6}{\pi^{2}} \sum_{i=1}^{n} \nu_{i} L\left(Q_{i}\right)=L_{A^{\prime}},
$$

where the value $L_{A^{\prime}}$ is the rational number given in Table 1 .

In addition, there are identities for 'type $T_{n}$ ' (tadpole type). We define the 'Cartan matrix' $C$ as almost the same as type $A_{n}$ except that the last diagonal entry is 1 , not 2 . Also the 
Table 1. The value $L_{A^{\prime}}$.

\begin{tabular}{c|cccccccccc}
\hline$R_{n}$ & $A_{n}$ & $B_{n}$ & $C_{n}$ & $D_{n}$ & $E_{6}$ & $E_{7}$ & $E_{8}$ & $F_{4}$ & $G_{2}$ & $T_{n}$ \\
\hline$L_{A}$ & $\frac{n(n+1)}{n+3}$ & $\frac{n(2 n-1)}{n+1}$ & $n$ & $n-1$ & $\frac{36}{7}$ & $\frac{63}{10}$ & $\frac{15}{2}$ & $\frac{36}{7}$ & 3 & $\frac{n(2 n+1)}{2 n+3}$ \\
\hline$L_{A^{\text {b }}}$ & $\frac{n(n+1)}{n+4}$ & $\frac{2 n(2 n-1)}{2 n+3}$ & $\frac{2 n(n+1)}{2 n+3}$ & $\frac{2(n-1) n}{2 n+1}$ & $\frac{24}{5}$ & 6 & $\frac{80}{11}$ & $\frac{24}{5}$ & $\frac{8}{3}$ & $\frac{n(2 n+1)}{2 n+4}$ \\
\hline
\end{tabular}

matrices $A$ and $A^{b}$ are defined by $a_{i j}=2 \min (i, j)$ and $a_{i j}^{b}=\min (i, j)$, respectively; the latter is the same matrix $A^{b}$ for type $C_{n}$. Then, $\left(A^{b}\right)^{-1}=C$ holds. We set $\nu_{i}=1$. Again, (3) should hold for the value $L_{A^{\prime}}$ in Table 1 , where $T_{n}$ is formally included as a member of $R_{n}$.

In [3] these identities were partially obtained and generally motivated by the Rogers-Ramanujan type identities arising from nilpotent affine Hecke algebras (Nil-DAHA), but only some of them are identified with the known identities.

The authors of [3] expected the connection between (3) and dilogarithm identities from some $Y$-systems (and cluster algebras behind them). In this note we answer this question affirmatively, and, in particular, we confirm all the identities in question. The note has considerable overlap with the paper by Lee [8], but it is written for a different purpose and in a different perspective.

\section{Dilogarithm identities for $Y$-systems of simply laced type}

Let us recall the following dilogarithm identities proved by cluster algebra method [9]. For $\ell=2$ see also [2].

Let $C$ be any Cartan matrix of simply laced type $R_{n}=A_{n}, D_{n}, E_{6}, E_{7}, E_{8}$, and let $\ell \geq 2$ be any integer (called the level). Let $Y_{m}^{(i)}(i=1, \ldots, n ; m=1, \ldots, \ell-1)$ be the unique real positive solution of the system of equations

$$
\left(Y_{m}^{(i)}\right)^{2}=\frac{\prod_{j=1}^{n}\left(1+Y_{m}^{(j)}\right)^{2 \delta_{i j}-c_{i j}}}{\left(1+Y_{m-1}^{(i)}-1\right)\left(1+Y_{m+1}^{(i)}-1\right)},
$$

where $Y_{0}^{(i)}-1=Y_{\ell}^{(i)}-1=0$.

Theorem 1 ([9, Corollay 1.9]). The following identity holds

$$
\frac{6}{\pi^{2}} \sum_{i=1}^{n} \sum_{m=1}^{\ell-1} L\left(\frac{Y_{m}^{(i)}}{1+Y_{m}^{(i)}}\right)=\frac{(\ell-1) n h}{h+\ell},
$$

where $h$ is the Coxeter number of type $R_{n}$, i.e., $n+1,2 n-2,12,18,30$ for $A_{n}, D_{n}, E_{6}, E_{7}$, $E_{8}$, respectively.

The system of equations (4) is called the level $\ell$ constant $Y$-system associated with the quantum affine Kac-Moody algebra of (untwisted) type $R_{n}^{(1)}$, which is a specialization of the corresponding (non-constant) $Y$-system. It is also known as the (constant) $Y$-system of $R_{n} \times A_{\ell-1}$. See [6] for more information.

We explain below that all the identities (3) in question are the ones in (5) for $\ell=2$, 3 , or their specializations. 
Table 2. The Langlands dual of affine type.

\begin{tabular}{c|ccccccccc}
\hline$R_{n}^{(1)}$ & $A_{n}^{(1)}$ & $B_{n}^{(1)}$ & $C_{n}^{(1)}$ & $D_{n}^{(1)}$ & $E_{6}^{(1)}$ & $E_{7}^{(1)}$ & $E_{8}^{(1)}$ & $F_{4}^{(1)}$ & $G_{2}^{(1)}$ \\
\hline$S_{m}^{(r)}$ & $A_{n}^{(1)}$ & $A_{2 n-1}^{(2)}$ & $D_{n+1}^{(2)}$ & $D_{n}^{(1)}$ & $E_{6}^{(1)}$ & $E_{7}^{(1)}$ & $E_{8}^{(1)}$ & $E_{6}^{(2)}$ & $D_{4}^{(3)}$ \\
\hline
\end{tabular}

\section{Identification with $\boldsymbol{Y}$-systems from quantum affine Kac-Moody algebras}

\subsection{The non-b case}

We consider the case $A^{\prime}=A$. We use the change of variables

$$
Q_{i}=\frac{Y_{i}}{1+Y_{i}}
$$

so that the range $0<Q_{i}<1$ corresponds to the range $0<Y_{i}$. Then, using (1), one can transform the equations (2) into the form

$$
\prod_{j=1}^{n}\left(\frac{1}{1+Y_{j}}\right)^{c_{i j}}=\left(\frac{Y_{i}}{1+Y_{i}}\right)^{2}
$$

which is equivalent to

$$
Y_{i}^{2}=\prod_{j=1}^{n}\left(1+Y_{j}\right)^{2 \delta_{i j}-c_{i j}}
$$

For simply laced types $A_{n}, D_{n}, E_{6}, E_{7}, E_{8},(7)$ coincides with the level 2 constant $Y$-system (4) of untwisted type $R_{n}^{(1)}$ by identifying $Y_{i}$ with $Y_{1}^{(i)}$. Then, the right hand side of (5) with $\ell=2$ gives the value of $L_{A}$, agreeing with Table 1 .

In contrast, for types $B_{n}, C_{n}, F_{4}, G_{2},(7)$ coincides with the level 2 constant $Y$-system of twisted type $S_{m}^{(r)}$ (in the sense of [6, Remark 9.22]), where $S_{m}^{(r)}$ is the Langlands dual of $R_{n}^{(1)}$. See Table 2 for the Langlands dual of affine type. Also see [6, Section 9] for the full version of $Y$-systems of twisted type. In this case, the direct inspection of the Cartan matrix shows that the equation (7) can be obtained from the level 2 constant $Y$-system of the untwisted (and simply laced) type $S_{m}^{(1)}$ by folding, i.e., identifying the variables with the diagram automorphism $\sigma$ of $S_{m}$. This is possible, due to the symmetry $Y_{m}^{(i)} \leftrightarrow Y_{m}^{(\sigma(i))}$ of the $Y$-system (4). Furthermore, it is easy to see that $\nu_{i}$ coincides with the number of elements in the $\sigma$-orbit of $i$. Thus, we obtain the identity (3) for type $R_{n}$ with $L_{A}\left(R_{n}\right)=L_{A}\left(S_{m}\right)$. For example, for $R_{n}=B_{n}$, $L_{A}\left(B_{n}\right)=L_{A}\left(A_{2 n-1}\right)$. This confirms and explains Table 1 .

Finally, for type $T_{n},(7)$ coincides with the level 2 constant $Y$-system of type $A_{2 n}^{(2)}$ (in the sense of [6, Remark 9.22]). Note that $A_{2 n}^{(2)}$ is self-dual under the Langlands duality. Again, this $Y$-system is obtained by the folding of level 2 constant $Y$-system of untwisted $A_{2 n}^{(1)}$. Since we set $\nu_{i}=1$, the multiplicities are discarded in (3). Therefore, $L_{A}\left(T_{n}\right)=L_{A}\left(A_{2 n}\right) / 2$. Actually, this connection is known in [3] and other literature.

\subsection{The $b$ case}

We consider the case $A^{\prime}=A^{b}$. By the same change of variables (6), one can transform the equations (2) into the form

$$
\prod_{j=1}^{n}\left(\frac{1}{1+Y_{j}}\right)^{c_{i j}}=\left(\frac{Y_{i}}{1+Y_{i}}\right)=\left(\frac{Y_{i}}{1+Y_{i}}\right)^{2}\left(1+Y_{i}^{-1}\right)
$$


which is equivalent to

$$
Y_{i}^{2}=\frac{\prod_{j=1}^{n}\left(1+Y_{j}\right)^{2 \delta_{i j}-c_{i j}}}{1+Y_{i}^{-1}} .
$$

For simply laced types, $A_{n}, D_{n}, E_{6}, E_{7}, E_{8},(8)$ is obtained from the level 3 constant $Y$ system (4) of untwisted type $R_{n}^{(1)}$ by the specialization $Y_{1}^{(i)}=Y_{2}^{(i)}$ and identifying it with $Y_{i}$. This is possible, due to the symmetry $Y_{1}^{(i)} \leftrightarrow Y_{2}^{(i)}$ of level $3 Y$-system (4). (One can also view it as the folding of $A_{2}$ to $T_{1}$ in the second component of $R_{n} \times A_{2}$.) Since we discard the multiplicity in (3), $L_{A^{b}}$ is the half of the right hand side of (5) with $\ell=3$. This agrees with Table 1.

Similarly, for the rest of types, (8) is obtained from the level 3 constant $Y$-system of type $S_{m}^{(r)}$ or $A_{2 n}^{(2)}$ (in the sense of [6, Remark 9.22]) by the specialization $Y_{1}^{(i)}=Y_{2}^{(i)}$, and the latter is further obtained from the level 3 constant $Y$-system of type $S_{m}^{(1)}$ or $A_{2 n}^{(1)}$ by the folding. Then, one can confirm Table 1.

Let us summarize the result.

Theorem 2. The identity (3) holds. Moreover, except for type $T_{n}$, the value $L_{A^{\prime}}$ in (3) has a unified expression

$$
L_{A^{\prime}}=\frac{m h^{*}}{h^{*}+\ell},
$$

where $\ell=2$ for $A^{\prime}=A$ and $\ell=3$ for $A^{\prime}=A^{b}$, and $m$ and $h^{*}$ are the rank and Coxeter number of $S_{m}$ for the Langlands dual $S_{m}^{(r)}$ of $R_{n}^{(1)}$.

We remark that the dilogarithm identities for untwisted and nonsimply laced types $B_{n}^{(1)}$, $C_{n}^{(1)}, F_{4}^{(1)}, G_{2}^{(1)}$ are also known $[4,5]$. It is natural to ask whether they will also appear from Nil-DAHA.

\section{Connection to cluster algebraic method}

For the reader's convenience, we include a brief explanation of the background of the dilogarithm identity (5), especially in the cluster algebraic method. See [7] and references therein for more information.

(a) $Y$-systems and dilogarithm identities. As the name suggests, the constant $Y$-system (4) is the constant version of the following (non-constant) $Y$-system

$$
Y_{m}^{(i)}(u+1) Y_{m}^{(i)}(u-1)=\frac{\prod_{j=1}^{n}\left(1+Y_{m}^{(j)}(u)\right)^{2 \delta_{i j}-c_{i j}}}{\left(1+Y_{m-1}^{(i)}(u)^{-1}\right)\left(1+Y_{m+1}^{(i)}(u)^{-1}\right)},
$$

where the variables $Y_{m}^{(i)}(u)$ now carry the spectral parameter $u \in \mathbb{C}$. The $Y$-system (9) appears in the thermodynamic Bethe ansatz (TBA) analysis of the deformation of conformal field theory. A constant solution $Y_{m}^{(i)}:=Y_{m}^{(i)}(u)$ of $(9)$, which is constant with respect to $u$, satisfies the constant $Y$-system (4), that also appears in the TBA analysis to calculate the effective central charge of conformal field theory.

The $Y$-system (9) has the following two remarkable properties.

(i) Periodicity

$$
Y_{m}^{(i)}(u+2(h+\ell))=Y_{m}^{(i)}(u) .
$$


(ii) Dilogarithm identity. For any positive real solution of (9), the following identity holds

$$
\frac{6}{\pi^{2}} \sum_{u=0}^{2(h+\ell)-1} \sum_{i=1}^{n} \sum_{m=1}^{\ell-1} L\left(\frac{Y_{m}^{(i)}(u)}{1+Y_{m}^{(i)}(u)}\right)=2(\ell-1) n h .
$$

The identity (5) is obtained by specializing the identity (11) to the (unique) positive real constant solution, and dividing the both hand sides of $(5)$ by the period $2(h+\ell)$.

(b) Cluster algebras and dilogarithm identities. It was once formidable to prove the properties (10) and (11) in full generality. However, they are now proved and rather well understood by the cluster algebraic method. In general, to any period of $y$-variables (coefficients) of a cluster algebra, the following dilogarithm identity is associated

$$
\frac{6}{\pi^{2}} \sum_{t=1}^{p} L\left(\frac{y_{k_{t}}(t)}{1+y_{k_{t}}(t)}\right)=N_{-}
$$

where $k_{1}, \ldots, k_{p}$ are the sequence of mutations for which $y$-variables are periodic, $y_{k_{t}}(t)$ is the $y$-variable mutated at $t$, and $N_{-}$is the total number of $t \in\{1, \ldots, p\}$ such that the tropical sign of $y_{k_{t}}(t)$ is minus. One can apply this general result to our $Y$-system (9). First, the $Y$-system is embedded into the $y$-variables of a certain cluster algebra. Then, by proving the periodicity of $y$-variables of this cluster algebra, we obtain the periodicity (10) of the $Y$-system. Finally, from the general identity (12), we obtain the dilogarithm identity (11) by calculating the constant term $N_{-}$.

(c) Quantum cluster algebras and quantum dilogarithm identities. One can lift the result in (b) to the quantum case. Namely, any period of $y$-variables of a cluster algebra can be lifted to the period of quantum y-variables of the corresponding quantum cluster algebra. Then, to any such period, the quantum dilogarithm identity is associated; furthermore, taking the semiclassical limit of the quantum dilogarithm identity we recover the classical dilogarithm identity (12). We expect that the Rogers-Ramanujan type identities of [3] are also deduced from these quantum dilogarithm identities. Some result in this direction is obtained by [1].

\section{Acknowledgements}

I thank Ivan Cherednik for raising this interesting question to me.

\section{References}

[1] Cecotti S., Neitzke A., Vafa C., R-twisting and 4d/2d correspondences, arXiv:1006.3435.

[2] Chapoton F., Functional identities for the Rogers dilogarithm associated to cluster $Y$-systems, Bull. London Math. Soc. 37 (2005), 755-760.

[3] Cherednik I., Feigin B., Rogers-Ramanujan type identities and Nil-DAHA, arXiv:1209.1978 (especially version 2).

[4] Inoue R., Iyama O., Keller B., Kuniba A., Nakanishi T., Periodicities of T- and $Y$-systems, dilogarithm identities, and cluster algebras I: Type $B_{r}$, Publ. Res. Inst. Math. Sci., to appear, arXiv:1001.1880.

[5] Inoue R., Iyama O., Keller B., Kuniba A., Nakanishi T., Periodicities of $T$ - and $Y$-systems, dilogarithm identities, and cluster algebras II: Types $C_{r}, F_{4}$, and $G_{2}$, Publ. Res. Inst. Math. Sci., to appear, arXiv:1001.1881.

[6] Inoue R., Iyama O., Kuniba A., Nakanishi T., Suzuki J., Periodicities of T-systems and Y-systems, Nagoya Math. J. 197 (2010), 59-174, arXiv:0812.0667.

[7] Kashaev R.M., Nakanishi T., Classical and quantum dilogarithm identities, SIGMA 7 (2011), 102, 29 pages, arXiv:1104.4630.

[8] Lee C.H., Nahm's conjecture and $Y$-system, arXiv:1109.3667.

[9] Nakanishi T., Dilogarithm identities for conformal field theories and cluster algebras: simply laced case, Nagoya Math. J. 202 (2011), 23-43, arXiv:0909.5480. 